\title{
Visually guided facial actions in rhesus monkeys
}

\author{
AKIHIRO IZUMI, KOJI KURAOKA, SHOZO KOJIMA, and KATSUKI NAKAMURA \\ Kyoto University, Inuyama, Japan
}

\begin{abstract}
We trained 2 monkeys to display facial actions in response to corresponding arbitrary visual cues. Each monkey executed the task successfully, and each displayed two different facial actions corresponding to either hand-sign or color cues. More than $90 \%$ of the responses were correct for each monkey. These results provide evidence that monkeys can execute facial actions in response to conditioned visual cues in the absence of social context. These data suggest that facial actions of monkeys are flexible enough for use in further laboratory investigations-for example, in studies on the neural mechanisms underlying the execution of actions.
\end{abstract}

In primates, large regions of the sensory and motor cortices enable the hands and face to process delicate sensations and execute precise actions (Penfield \& Rasmussen, 1950; Sherrington, 1947; Woolsey, 1958). Fine control of the hands enables the manipulation of objects, and that of the face enables the display of facial expressions and the production of voice. The neural mechanisms underlying the execution of manipulative actions in monkeys have been investigated extensively (Mountcastle, Lynch, Georgopoulos, Sakata, \& Acuna, 1975; Rizzolatti et al., 1988; Taira, Mine, Georgopoulos, Murata, \& Sakata, 1990), but those related to the execution of facial actions remain unclear. One possible reason why researchers have not studied the production of facial actions in monkeys is that the control of animal behavior entails difficulties. Monkeys, like humans, have welldeveloped facial muscles and display a rich variety of facial expressions for social communication (Darwin, 1872; Hauser, 1996; Redican, 1975; Van Hoof, 1962). Previously, the neural mechanisms underlying the perception of facial expressions have been studied (Hasselmo, Rolls, \& Baylis, 1989; Nakamura, Mikami, \& Kubota, 1992; Sugase, Yamane, Ueno, \& Kawano, 1999). Yet although monkeys can acquire the skills to perform various laboratory tasks such as the manipulation of objects, training them to perform facial tasks can be difficult. Facial expressions are signals for social communication, and such signals may not be expressed in the absence of social context. Furthermore, one cannot usually see one's own facial expressions, and this lack of visual feedback may be an obstacle to executing facial tasks.

This research was supported by HFSP project (principal investigator V. Gallese) and Grants-in-Aid for Scientific Research on Priority Areas (No. 12210092) and for Encouragement of Young Scientists (No. 12780597) from the Ministry of Education, Science, Sports, and Culture of Japan. Correspondence concerning this article should be addressed to K. Nakamura, Department of Behavioral and Brain Sciences, Primate Research Institute, Kyoto University, Inuyama, Aichi 4848506, Japan. (e-mail: knakamur@ pri.kyoto-uac.jp).
In this study, we trained monkeys to display facial actions in response to visual cues. Each monkey was trained to execute two different facial actions, and each action corresponded to two types of visual cues. The correspondence of visual cues to facial actions was arbitrary, and the task was independent of social context. We hoped that the successful acquisition of the skills for this task might enable more detailed studies of the neural mechanisms underlying the execution of facial actions.

\section{METHOD}

\section{Subjects}

Two male rhesus monkeys (Macaca mulatta) served as subjects (Dai, 5 years old, $6.0 \mathrm{~kg}$; Tiga, 4 years old, $4.5 \mathrm{~kg}$ ). Water was withheld before each daily session but was given as a reward during the experiment. Following the daily sessions, the monkeys were returned to their individual cages and supplemental water and vegetables were provided as needed. All husbandry and experimental procedures were in accordance with the Guide for the Care and Use of Laboratory Animals of the National Research Council (1996) and the Guide for the Care and Use of Laboratory Primates of the Primate Research Institute, Kyoto University (1986).

\section{Experimental Setup}

All of the experiments were performed in an experimental room. The monkey and a human observer were in the room, separated by a curtain. The monkey was seated in a primate chair facing a 17-in. computer display, which was $55 \mathrm{~cm}$ away from the subject. Visual cues were presented on the display. A video camera monitoring the monkey's facial actions was set beside the display and was connected to a video monitor facing the observer. Two personal computers controlled the task events and data collection. A movable pipe for delivering water as the reward was placed in front of the monkey, but it was too far away for the monkey to access it. After a correct response, the pipe was automatically moved toward the monkey, propelled by a motor.

\section{Facial Actions and Visual Stimuli}

The monkeys were required to display two different facial actions in response to visual cues. The predetermined correspondences between facial actions and visual cues are shown in Figure 1. Two actions for each monkey were selected on the basis of the high frequency of spontaneous execution before the training. One monkey (Dai) was trained to execute the actions of tongue protrusion and 
Dai

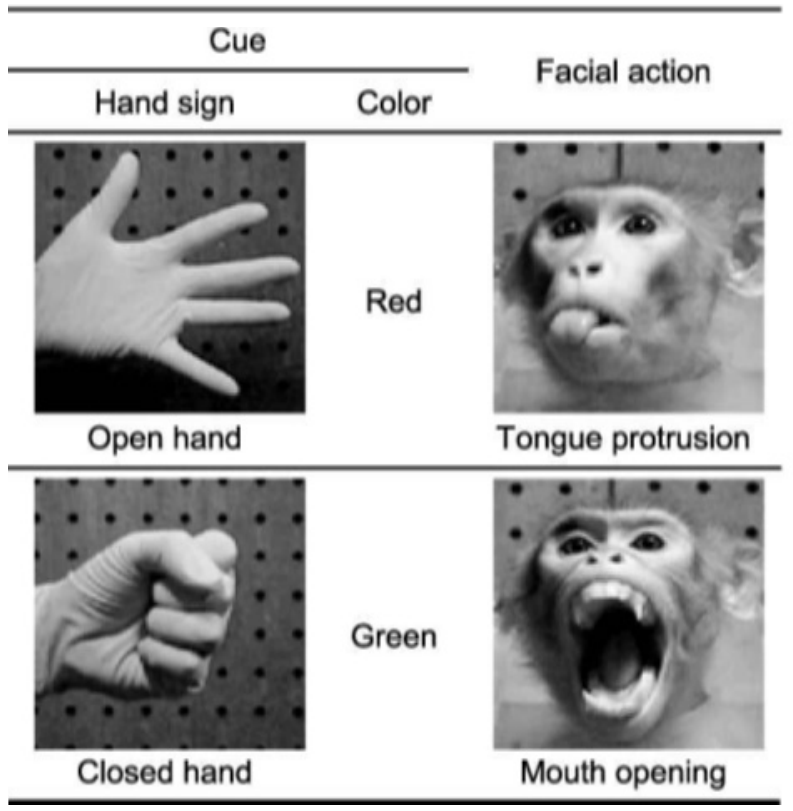

Tiga

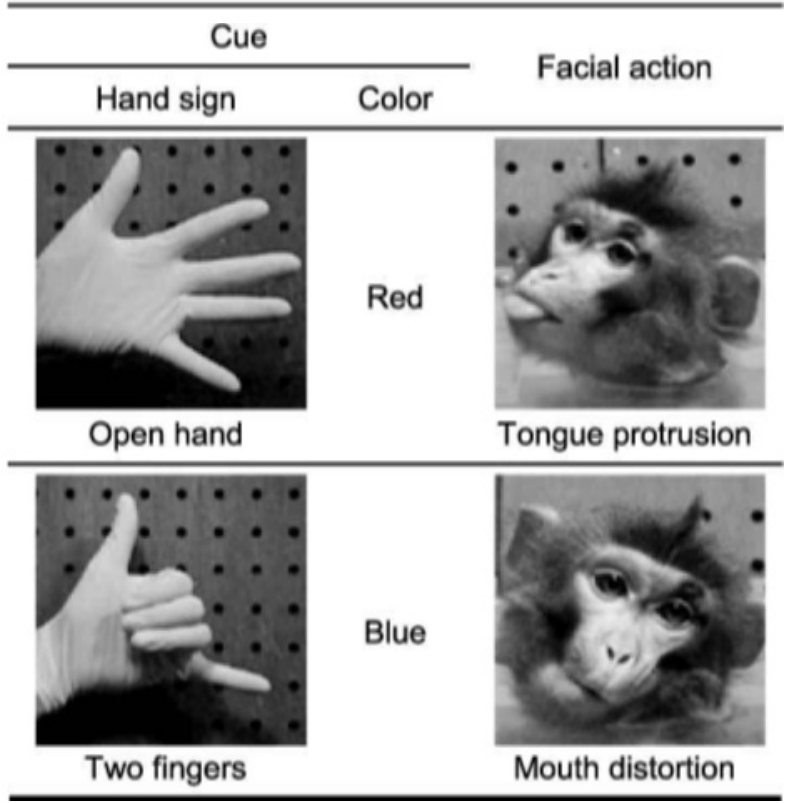

Figure 1. Target facial actions and corresponding visual cues. Each monkey was trained to perform two target actions: tongue protrusion and mouth opening for Dai, and tongue protrusion and mouth distortion for Tiga.

mouth opening, and the other monkey (Tiga) was trained to execute the actions of tongue protrusion and mouth distortion. Each trained action corresponded to two types of visual cues: a hand-sign cue and a color cue. Three stimuli were prepared for each type of visual cue. Therefore, a total of six visual cues was used: Open hand, closed hand, and two fingers were used as the hand-sign cues; and red, green, and blue were used as the color cues. A rectangular space $(21 \mathrm{~cm}$ wide $\times 14.5 \mathrm{~cm}$ high $)$ at the center of the display was used for the hand-sign cues, and the outer space was for the color cues. This allocation of space was for the simultaneous presentation of hand-sign and color cues used in one of the training steps. The hand-sign cues were in the format of video recordings of hand signs and were displayed on the computer monitor $(720 \times 480$ pixels). For color cues, the corresponding space of the monitor was filled with one of the colors; the space for hand-sign cues remained black.

Visual cues were introduced step-by-step in the training. Initially, a human experimenter stood beside the monkey and presented real hand signs as cues (i.e., manual cues). The cues were then replaced by the digital video recordings of the same hand signs. Following the acquisition of this training step with hand-sign cues, color cues were introduced. Initially, the color cues were presented simultaneously with hand-sign cues to allow the monkey to use both cues, and they were then used independently. When the monkey executed more than $90 \%$ of correct responses for either hand-sign or color cues, test sessions started.

\section{Assessment of Facial Actions}

There were two human observers who had trained the monkeys since the beginning of this study, and one of them participated in each session. The observer monitored the monkey's facial actions through the video monitor that was connected to the video camera monitoring the monkey's face. The observer could not see the visual cues presented on the display.

To confirm the validity of the facial assessment, we examined the assessment by the two observers and by two extra observers who did not know this experiment in detail. They initially watched a sample of the video-recorded action for each of the two actions by the 2 monkeys, and they were required to classify 40 video-

Table 1

Individual Number of Responses for Each Stimulus Condition

\begin{tabular}{clccc}
\hline \multirow{2}{*}{ Subject } & \multicolumn{1}{c}{ Cue } & \multicolumn{3}{c}{ Response } \\
\cline { 3 - 5 } & Match $(\%)$ & Nonmatch & Other \\
\hline \multirow{2}{*}{ Dai } & Hand sign: open hand & $272(98 \%)$ & 5 & 0 \\
& Hand sign: closed hand & $315(98 \%)$ & 8 & 0 \\
& Color: red & $304(98 \%)$ & 7 & 0 \\
& Color: green & $285(99 \%)$ & 4 & 0 \\
Tiga & Hand sign: open hand & $301(94 \%)$ & 7 & 11 \\
& Hand sign: two fingers & $272(90 \%)$ & 21 & 10 \\
& Color: red & $283(93 \%)$ & 12 & 10 \\
& Color: blue & $256(94 \%)$ & 5 & 12 \\
\hline
\end{tabular}

Note-Data are from 10 sessions for each monkey. A session consisted of 120 trials, and the cumulative number of responses from each monkey was 1,200. The proportion of "match" responses for each stimulus type is shown in parentheses. 
recorded facial actions, including 20 actions for each of the 2 monkeys. The three observers agreed on their classification perfectly, and the other observer classified differently for only one facial action by Tiga. The high consistency among the observers confirmed the validity of the assessment in the present experiment.

\section{Behavioral Task}

At the beginning of a trial, a beep tone was sounded $1 \mathrm{sec}$ before the presentation of a visual cue to call the monkey's attention to the display. The visual cue was then presented on the display, and the monkey was required to execute the trained action corresponding to this cue within a $10-\mathrm{sec}$ stimulus presentation period. The human observer classif ied each facial action of the monkey during the stimulus presentation period. The observer was required to press one of two switches to indicate the assessment that was transmitted to the computer. An appropriate facial action that corresponded to the visual cue was recorded as a match response. When the subject displayed the other trained action, it was recorded as a nonmatch response. A response was recorded as other when it was classified as neither match nor nonmatch (i.e., there was no response, the monkey displayed an untrained action, or the monkey displayed a trained facial action before the stimulus presentation). In the case of other responses, the observer pressed neither of the switches throughout the stimulus presentation period. Following a match response, the water pipe automatically moved toward the monkey's mouth, and a sip of water was supplied as a reward. Neither nonmatch nor other responses were rewarded. Each trial was followed by an intertrial interval (ITI) of $3 \mathrm{sec}$ during which time the display was darkened, and the next trial was started after the ITI. In each trial, a visual cue was selected randomly from four visual cues (two hand-sign and two color cues were intermingled).

We calculated the response latencies in two training sessions just before the test sessions. The latencies for Dai and Tiga were $1.07 \pm$ $0.47 \mathrm{sec}$ and $1.57 \pm 0.94 \mathrm{sec}$ (average $\pm S D$ ), respectively, and the 10 -sec observation period was long enough for the monkeys to execute facial actions. Further analysis of the reaction times was not undertaken, because the reaction time included the time in which the human observer assessed the facial actions and pressed one of the switches.

A test session consisted of 120 trials, and each monkey had one session per day. If the other responses exceeded $10 \%$ of the trials in a session, the data from that session were discarded. In the case of the monkey Tiga, other responses exceeded $10 \%$ of the trials in three sessions, probably because of low motivation, and so the data were not included. Each monkey was required to complete 10 valid sessions.

\section{RESULTS}

Before the beginning of the test sessions, both monkeys required about 80 training sessions, including 50 sessions with manual cues. The monkeys showed quick transfer from the real hand-sign cues to the video-recorded cues. In the case of the introduction of color cues, more extensive training was required.

In the test sessions, each monkey performed more than 250 trials under each stimulus condition. The task performances of the 2 monkeys are summarized in Table 1. Dai executed more than $97 \%$ of match actions in response to all four stimuli, and executed no other actions throughout the experimental sessions. Tiga also displayed a high performance. Under all four stimulus conditions, more than $90 \%$ were match actions. However, unlike Dai, Tiga sometimes executed other actions (10-12 trials, 3-5\%).
Most other responses were no responses. For both monkeys, the frequency of match responses was significantly higher than that of nonmatch responses for each stimulus condition (binomial test, one-tailed $p<.01$ ). The observer was not aware of which action was a match response when assessing the monkey's facial actions. Thus, no bias of the observer affected the results. These data indicate that the monkeys accurately displayed two facial actions in response to visual cues, irrespective of the type of cue.

\section{DISCUSSION}

The objective of this study has been to examine whether monkeys' facial actions are flexible enough for use in further laboratory investigations. That is, whether monkeys can be trained to execute facial actions under conditions in which the facial actions are isolated from social context and triggered by conditioned visual stimuli. The 2 monkeys successfully executed the trained facial actions in response to corresponding visual cues. Although the hand-sign cues may have some social meanings and therefore might have triggered an emotional response, it is unlikely that the colored computer graphics had social meanings for the monkeys. Therefore, our results suggest that monkeys can execute facial actions in response to corresponding conditioned visual cues in the absence of social context if the monkeys are appropriately trained. Unlike with other manipulations, one does not usually see one's own facial actions. Although it is not clear what mediated the cue-action relationship in the present facial action task, visual feedback regarding the monkeys' own faces was not necessary for the monkeys to acquire the task.

We successfully trained 2 monkeys to perform two different facial actions. This does not mean, however, that monkeys can be trained to display any facial action. The trained actions were ones that the monkeys had executed spontaneously and frequently before the training. Some monkeys can be trained to open their mouths, but others cannot. However, we successfully trained both monkeys to execute tongue protrusion, which is closely related to licking. Actions such as this, which are frequently observed in all monkeys, can be trained easily. Monkeys have less chance to learn the relationship between an action and reinforcement if they show the action infrequently.

We conclude that monkeys' facial actions are flexible enough for use in further investigations. For example, it might be possible to investigate the neural bases of facial actions just as the neural bases of manipulation have been investigated. Although the monkeys could display facial actions in the absence of a social context, social cues may still affect such conditioned facial actions. Some interactions can occur between emotional sensory inputs and signals associated with the execution of facial actions. For example, reconcilable vocalizations of monkeys may inhibit displays of the mouth opening because this facial action has an aggressive connotation. The 
modified application of the present methods may be useful in future studies on the mechanisms of the neural correlates of emotional behavior.

\section{REFERENCES}

DARWIN, C. (1872). The expression of the emotion in man and animals. London: John Murray.

Hasselmo, M. E., Rolls, E. T., \& Baylis, G. C. (1989). The roles of expression and identity in the face-selective responses of neurons in the temporal visual cortex of the monkey. Behavioural Brain Research, 32, 203-218.

HAUSER, M. D. (1996). The evolution of communication. Cambridge, MA: MIT Press.

Mountcastle, V. B., Lynch, J. C., Georgopoulos, A., Sakata, H. \& ACUNA, C. (1975). Posterior parietal association cortex of the monkey: Command functions for operations within extrapersonal space. Journal of Neurophysiology, 38, 871-908.

Nakamura, K., Mikami, A., \& Kubota, K. (1992). Activity of single neurons in the monkey amygdala during performance of a visual discrimination task. Journal of Neurophysiology, 67, 1447-1463.

Penfield, W., \& Rasmussen, T. (1950). The cerebral cortex of man: A clinical study of localization of function. New York: Macmillan.

REDICAN, W. K. (1975). Facial expressions in nonhuman primates. In
L. A. Rosenblum(Ed.), Primate behavior (Vol. 4, pp. 103-194). New York: Academic Press.

Rizzolatti, G., Camarda, R., Fogassi, L., Gentilucci, M., LupPINO, G., \& MATELLI, M. (1988). Functional organization of inferior area 6 in the macaque monkey: II. Area F5 and the control of distal movements. Experimental Brain Research, 71, 491-507.

SHERRINGTON, C. S. (1947). The integrative action of the nervous system (2nd ed.). New Haven, CT: Yale University Press.

Sugase, Y., Yamane, S., Ueno, S., \& Kawano, K. (1999). Global and fine information coded by single neurons in the temporal visual cortex. Nature, 400, 869-873.

Taira, M., Mine, S., Georgopoulos, A. P., Murata, A., \& Sakata, H. (1990). Parietal cortex neurons of the monkey related to the visual guidance of hand movements. Experimental Brain Research, 83, 29-36.

VAN Hoof, J.A.R.A.M. (1962). Facial expressions in higher primates. Symposia of the Zoological Society of London, 8, 97-125.

Woolsey, C. N. (1958). Organization of somatic sensory and motor areas of the cerebral cortex. In: H.F. Harlow \& C. N. Woolsey (Eds.), Biological and biochemical bases of behavior (pp. 63-81). Madison: University of Wisconsin Press.

(Manuscript received January 17, 2001;

revision accepted for publication June 21,2001 .) 